\title{
Multiplication simultanée de plusieurs virus à ADN chez les Lépidoptères
}

\author{
par F. ODIER \\ Station de Recherches de Cytopathologie, I.N.R.A.-C.N.R.S., F 30380 Saint-Christol
}

A côté des problèmes d'immunité naturelle vis-à-vis des virus et de la protection passagère observée après un traitement préparatoire, on envisage simultanément la mise en évidence de phénomènes de tolérance et la coexistence de plusieurs viroses car ces aspects rejoignent la voie de l'immunologie comparée. En effet, chez les Invertébrés, la présence simultanée et les interactions de plusieurs germes pathogènes semblent être des processus très répandus.

Nos recherches s'insèrent dans ces préoccupations en proposant l'étude expérimentale des syndromes et des actions pathologiques complexes se greffant sur ce type de maladie donnée: la virose de type densonucléose (Vago et al., 1964). C'est ainsi que nous avons étudié, chez le lépidoptère Galleria mellonella, la double infection à Parvovirus et Baculovirus à Parvovirus et Iridovirus et la triple infection à Parvovirus, Baculovirus et Iridovirus. Le choix de ces complexes a été motivé par l'intérêt que présente en virologie comparée l'étude des interactions de trois virus à $\mathrm{ADN}$ dont deux ont une pathogénèse nucléaire (virus de la densonucléose et de la polyédrose nucléaire) (Amargier et al., 1968) et le troisième un développement cytoplasmique (virus irisant de Chilo suppressalis) (Kurstak et al., 1972). Pour chacun des complexes cités, nous avons étudié les infections simultanées et décalées dans le temps pour suivre l'influence du développement de chacune des viroses dans le temps pour suivre l'influence du développement de chacune des viroses sur les autres membres du complexe. L'orientation de ces études est essentiellement histologique et cytologique (Odier, 1974).

Complexe à Parvovirus (virus de la densonucléose) et Baculovirus (virus de la polyédrose nucléaire de Galleria).

Les lésions histologiques et cytologiques caractérisant la présence de chacune des viroses dans les cellules séparées d'un même tissu ont été mises en évidence. 
Dans d'autres cas, les signes de la double pathogénèse apparaissent dans un même noyau où on note l'abondance de virions de la densonucléose à côté de plages virogènes de Baculovirus. Les deux synthèses virales peuvent donc avoir lieu dans un même noyau. Cependant, des signes de l'influence du développement de l'un des virus sur l'autre ont pu être déterminés. On a pu ainsi noter des anomalies dans la formation des virions de Baculovirus, la présence de microtubules et surtout le nombre réduit des virions inclus dans les polyèdres.

Complexe à Parvovirus (virus de la densonucléose) et Iridovidus (virus irisant de Chilo suppressalis).

Les infections dues à ce complexe permettent de constater le développement des virions du Parvovirus dans le noyau et des virions du virus irisant dans le cytoplasme, soit dans des tissus différents, soit dans des cellules différentes d'un même tissu, soit dans une même cellule.

Ici, il est à noter le caractère particulièrement envahissant de l'Iridovirus par rapport aux lésions dues au Parvovirus.

\section{Complexe à Parvovirus, Baculovirus et Iridovirus.}

Il est probable que le paramètre représenté par la variation des titres des inoculums est susceptible d'introduire des différences dans l'évolution des processus pathologiques. C'est pourquoi, nous avons fait varier la concentration en virus de chacune des suspensions afin de savoir s'il existe une prédominance de l'un des virus sur l'autre. Les lésions histologiques et cytologiques se développant à la suite d'une triple infection montrent que les trois viroses sont susceptibles de s'installer simultanément dans un même individu et un même tissu. Le plus souvent, ce sont les lésions à Baculovirus et Iridovirus qui sont les plus importantes et ceci quelle que soit la concentration en virus de trois suspensions.

\section{Les complexes en tant que syndromes transmissibles.}

Les trois types de complexes étudiés ont été transmis comme tels à des larves saines par ingestion de cadavres de larves atteintes de ces mêmes complexes. Nous avons observé alors les lésions histologiques et cytologiques caractéristiques de chacune des viroses (Odier, 1975).

L'ensemble de ces observations montre que les complexes de maladies étudiés peuvent se dérouler dans un même individu, un même tissu et une même cellule. La tolérance de la cellule d'insecte vis-à-vis des infections complexes parait être remarquable mais les anomalies enregistrées n'excluent pas un processus d'inhibition de faible intensité.

Sur le plan écologique, cette tolérance peut se traduire par la transmission d'un individu à l'autre ou d'une population à l'autre des complexes de viroses en tant que syndromes relativement stables. 


\section{Bibliographie}

Amargier (A.), Meynadier (G.) et Vago (C.), 1968. - Un complexe de viroses: polyédrie nucléaire et densonucléose chez le Lépidoptère Galleria mellonella L. Mikroskopie, 23, 244-251.

Kurstak (E.), Garzon (S.) et ONJi (P.-A.), 1972. - Replication simultanée de deux virus à DNA. Microscopie à fluorescence et électronique. Arch. ges. Virusforsch., 36, 324-334.

Odier (F.), 1974. - Recherches sur les interactions et les complexes de maladies liés à des viroses de type densonucléose chez les Lépidoptères. Thèse Doctorat d'Etat, Univ. Sciences, Montpellier.

Odier (F.), 1975. - Les complexes de viroses: entités pathologiques transmissibles chez les Invertébrés. C.R. Acad. Sci., D, 280, 2277-2280.

Vago (C.), Meynadier (G.) et Duthort (J.-L.), 1964. - Etude d'un nouveau type de maladie à virus chez les Lépidoptères. Ann. Epiphyties, 15, 473-477. 